\title{
THE SPECTRAL INDEX DISTRIBUTION OF EGRET BLAZARS: PROSPECTS FOR GLAST
}

\author{
TONia M. Venters ${ }^{1}$ \& VASILIKI PAVlidou ${ }^{2,3}$ \\ Draft version February 5, 2008
}

\begin{abstract}
The intrinsic distribution of spectral indices in GeV energies of gamma-ray-loud blazars is a critical input in determining the spectral shape of the unresolved blazar contribution to the diffuse extragalactic gamma-ray background, as well as an important test of blazar emission theories. We present a maximum-likelihood method of determining the intrinsic spectral index distribution (ISID) of a population of $\gamma$-ray emitters which accounts for error in measurement of individual spectral indices, and we apply it to EGRET blazars. We find that the most likely Gaussian ISID for EGRET blazars has a mean of 2.27 and a standard deviation of 0.20 . We additionally find some indication that FSRQs and BL Lacs may have different ISIDs (with BL Lacs being harder). We also test for spectral index hardening associated with blazar variability for which we find no evidence. Finally, we produce simulated GLAST spectral index datasets and perform the same analyses. With improved statistics due to the much larger number of resolvable blazars, GLAST data will help us determine the ISIDs with much improved accuracy. Should any difference exist between the ISIDs of BL Lacs and FSRQs or between the ISIDs of blazars in the quiescent and flaring states, GLAST data will be adequate to separate these ISIDs at a significance better than $3 \sigma$.
\end{abstract}

Subject headings: galaxies: active - gamma rays: observations - gamma rays: theory

\section{INTRODUCTION}

The Energetic Gamma-Ray Experiment Telescope (EGRET) aboard the Compton Gamma-Ray Observatory observed the gamma-ray sky in energies between $30 \mathrm{MeV}$ and $\sim 10 \mathrm{GeV}$ between 1991 and 2000. The third (and last) catalog of EGRET point sources (Hartmann et al. 1999) included 271 resolved objects of which 93 were identified, either confidently or potentially, as blazars (gamma-ray loud active galactic nuclei). Thus, blazars constitute the class of $\gamma$-ray emitters with the largest number of identified members.

The term "blazar" is used to refer collectively to BL Lac objects and $\gamma$-ray loud flat spectrum radio quasars (FSRQs). Blazars are believed to be active galactic nuclei (AGNs) with the jet aligned with our line-of-sight (Blandford \& Königl 1979; see Urry \& Padovani 1995 for a review of general properties of blazars). The main contribution to the $\gamma$-ray emission of blazars is generally thought to result from either inverse Compton scattering by relativistic electrons in the jet of lower energy photons, produced either by synchrotron emission within the jet (SSC), or by emission external to the jet, for example from the accretion disk around the central engine (EC); or from proton-induced cascades (see, e.g., von Montigny et al. 1995, Böttcher 2006 and references therein for a review of blazar $\gamma$-ray emission processes).

Blazars fainter than the ones in the 3rd EGRET (3EG) catalog (and thus unresolved by EGRET) are expected to have a sizable contribution to the diffuse, isotropic gamma-ray background detected by EGRET (Sreekumar et al. 1998) which is presumably of extragalactic origin. The exact amount of this contribution remains unclear,

\footnotetext{
${ }^{1}$ Department of Astronomy and Astrophysics, The University of Chicago, Chicago, IL 60637

2 Kavli Institute of Cosmological Physics, The University of Chicago, Chicago, IL 60637

3 Enrico Fermi Institute, The University of Chicago, Chicago, IL 60637
}

as different models for the blazar $\gamma$-ray luminosity function result in very different predictions for the cumulative $\gamma$-ray flux from all unresolved blazars, ranging from a few to $100 \%$ of the EGRB (Padovani et al. 1993; Stecker et al. 1993; Salamon \& Stecker 1994; Chiang et al. 1995; Stecker \& Salamon 1996a, hereafter SS96a; Kazanas \& Perlman 1997; Chiang \& Mukherjee 1998; Mukherjee \& Chiang 1999; Mücke \& Pohl 2000; Kneiske \& Mannheim 2005; Dermer 2007; Giommi et al. 2006; Narumoto \& Totani 2006). Such uncertainties aside, blazars must be accounted for in any attempt to understand, model, or otherwise make use of the high-energy diffuse photon inventory (e.g. in constraining exotic high-energy physics). The lowest reasonable value we can expect for the cumulative diffuse intensity of unresolved faint blazars yields a strong lower limit for the level of the extragalactic gamma-ray background (EGRB). To constrain contributions from any other plausible source, this lower limit must be subtracted from the observed background.

A second critical property of blazars is their spectral index distribution (SID). The energy spectrum of the $\gamma$ ray emission of blazars in the EGRET energy range can be well approximated by a power law, $F(E) \propto E^{-\alpha}$ with values of $\alpha$, the spectral index, for individual blazars fitted to be in most cases between 2 and 3. Blazar spectra in the $\gamma$-ray regime encode important information concerning particle acceleration and emission processes in blazar jets (see von Montigny et al. 1995 and references therein). In addition, the distribution of blazar $\gamma$-ray spectral indices is a critical input in the estimation of the unresolved blazar contribution to the extragalactic diffuse background (SS96a; Pohl et al. 1997). Whether the collective emission of unresolved blazars may be the dominant component of the diffuse extragalactic gammaray background depends not only on intensity, but also on spectral shape (Stecker \& Salamon 1996a,b; Pohl et al. 1997; Strong et al. 2004). Additionally, blazars may constitute the dominant component of the extragalactic 
gamma-ray background only at certain energies (Pavlidou \& Fields 2002). In order to assess these issues, the spectral features of the unresolved blazar emission need to be studied, and hence the spectral index distribution of blazars needs to be understood.

\subsection{Past Work}

Obtaining the spectral index distribution of blazars presents three major difficulties:

(1) Large measurement uncertainties in individual blazar spectral indices, due to low photon statistics. These errors contaminate the sampling of the underlying intrinsic spectral index distribution (ISID) ${ }^{4}$, by exaggerating its spread, possibly to a large degree.

(2) Possible systematic change of the spectral index with flaring [suggested, e.g., by von Montigny et al. 1995; Mukherjee et al. 1996 (however, note that for the case of PKS $0528+134$, the significance of the result was reduced by subsequently obtained data, see Mukherjee et al. 1997); SS96a; Mücke et al. 1996; Pohl et al. 1997].

(3) Possible existence of two spectrally distinct populations (BL Lacs and FSRQs) in the resolved blazar sample (e.g. Mukherjee et al. 1997; Pohl et al. 1997)

Despite these difficulties, several authors have studied different aspects of the statistical properties of $\mathrm{GeV}$ blazar spectra. SS96a calculated a spectral index distribution for resolved EGRET blazars which they then used to derive the spectral shape of the collective emission from unresolved blazars. They recognized that an appreciable spread in blazar spectral indices will lead to a pronounced concavity in the collective emission spectrum (see Brecher \& Burbidge 1972), a feature which is also at least tentatively present in determinations of the EGRB based on EGRET data (Sreekumar et al. 1998; Strong et al. 2004). Additionally, they insightfully emphasized the potential importance of both variability as well as measurement errors in the determination of the blazar SID. SS96a remains to this day the only work associating the distribution of blazar spectral indices as measured in individual objects with a model predicting the spectral shape of the unresolved blazar emission.

However, their treatment suffers from three major unresolved problems. First, their treatment of variability was based on very uncertain information from very few objects. Second, BL Lacs and FSRQs are treated as a single population, while the validity of this assumption was not evaluated. Finally, their treatment of measurement errors worsens, rather than alleviates, the overestimation of the SID spread, and thus, overestimates the curvature of the unresolved blazar emission spectrum. This problem will not automatically disappear when the much larger sample of detected blazars and corresponding measured spectral indices of the upcoming GLAST mission becomes available. The bulk number of blazar detections will always be close to the instrument sensitivity limit, and will involve only a few tens of photons from each object, which means that the bulk number of measured spectral indices will always have substantial measurement errors associated with them. Therefore,

\footnotetext{
${ }^{4}$ In this paper, we will use the term "spectral index distribution" (SID) to refer to the distribution of measured spectral indices and the term "intrinsic spectral index distribution" (ISID) to refer to the true distribution of spectral indices of the blazar population (i.e. free of any contamination due to measurement errors).
}

in order to be able to utilize all future measurements, including those for faint objects, in understanding the spectral properties of $\gamma$-ray-loud AGN in the GeV energy range, spectral index uncertainties have to be carefully dealt with.

Pohl et al. (1997) used a different method to derive the spectral shape of the collective unresolved emission of blazars, which effectively circumvents the problem of large uncertainties in the measurement of spectral indices of individual objects. They co-added the spectra of resolved AGN and pointed out that if the spectral properties of unresolved blazars are similar to those of resolved blazars, then this co-added spectrum is the most appropriate quantity (in terms of spectral shape) for comparison with observations of the EGRB. They performed the analysis separately for BL Lacs and FSRQs, and found that the BL Lac co-added spectrum is harder by $\delta \alpha=0.12 \pm 0.08$ than that of FSRQs, although the significance of the difference is low due to the low-number BL Lac statistics. Similarly, they found a difference between the FSRQ co-added flaring spectrum and the time-averaged co-added FSRQ spectrum, with the flaring spectrum being harder by $\delta \alpha=0.18 \pm 0.05$, a result with a statistical significance between 3 and $4 \sigma$. They verified that the co-added spectra are concave, although their method yields a much smaller spectrum curvature than the SS96a model.

Mukherjee et al. (1997), as part of a comprehensive analysis of the EGRET blazar observations available at that time, evaluated the average spectral properties of the blazar population and tested for indications of evolution of the spectral index with redshift and spectral differences between FSRQs and BL Lacs. Assuming equal errors in individual measurements of spectral indices, they found an average spectral index of $2.15 \pm 0.04$ for all blazars, and average spectral indices of $2.03 \pm 0.09$ and $2.20 \pm 0.05$ for the subsets of BL Lacs and FSRQs, respectively. They concluded that the statistical significance of the difference between the mean spectral indices of the two populations was at the level of $2.5 \sigma$ and was not enough to justify a spectral separation of the two populations. Finally, they tested for redshift evolution of blazar spectra and found that for both BL Lacs and FSRQs, the data were consistent with no evolution. Neither Pohl et al. (1997) nor Muhkerjee et al. (1997) derived an SID for blazars.

\subsection{This Work}

The Gamma-ray Large Area Space Telescope (GLAST), which is scheduled for launch in 2007, will be able to address and ameliorate these difficulties. It will detect many more blazars than EGRET (between 1,000 and 10,000 blazars; Stecker \& Salamon 1999; Narumoto \& Totani 2006; Dermer 2007), enabling confirmation or rejection of any statistical trends seen in EGRET data. In order to facilitate the spectral studies of blazars with GLAST, it is important to use EGRET data to identify open questions and issues which can benefit from the dramatically improved GLAST statistics. Furthermore, it is advantageous to use EGRET data to identify and test appropriate statistical techniques which will allow us to deal with observational difficulties which are also expected to be present in GLAST data (such as large, varying errors of 
measurement in individual spectral indices).

In this context, with this paper we attempt to: (a) assess whether there is statistically significant evidence for evolutionary effects on the spectral index, manifesting as a correlation between the spectral index with either redshift or (isotropic) photon luminosity (\$2); (b) estimate the extent to which individual measurement errors affect the sampling of the SID of EGRET blazars (\$3) and perform a maximum likelihood analysis which accounts for these errors and determines the "most likely" parameters of the ISID (44); (c) assess whether there is statistically significant evidence for a difference between the ISIDs of BL Lacs and FSRQs (95) and the ISIDs of flaring and quiescent blazars (\$6); (d) predict to what extent the upcoming GLAST observations will help us in resolving the issues mentioned above (\$7). We summarize and discuss our conclusions in 8 .

The dataset used for our analysis, except where explicitly stated otherwise, is the set of 66 blazars characterized as "confident AGN identifications" in the 3EG catalog. We divide the population into 14 BL Lacs and 51 FSRQs (four sources have not been used due to lack of measured P1234 fluxes) as in Nolan et al. (2003). The spectral indices of individual objects are the average, P1234 indices quoted in $3 \mathrm{EG}$.

\section{DOES THE BLAZAR SID DEPEND ON REDSHIFT OR LUMINOSITY?}

If blazars evolve spectrally with redshift, or if their spectral properties depend on luminosity, then the SID calculated from resolved, low- $z$ and/or high-luminosity blazars is not representative of the spectral properties of unresolved, high- $z$ and/or low-luminosity blazars, and should not be used to calculate the spectral shape of their collective emission. For this reason, we test for correlations between blazar spectral index and redshift or luminosity. Figure 1 shows plots of the spectral index versus photon luminosity (upper panel) and redshift (lower panel) for the 66 3EG confident blazars. The (isotropic) photon luminosity $L_{p}$ (shown in Fig. 1 in units of $10^{50}$ photons/sec) was calculated from the P1234 photon flux $F_{P 1234}$ using

$$
L_{p}=4 \pi F_{P 1234} d_{L}^{2} /(1+z) .
$$

Here $d_{L}$ is the luminosity distance, calculated from $z$ assuming a concordance $\Omega_{\mathrm{m}}=0.3, \Omega_{\Lambda}=0.7, H_{0}=$ $70 \mathrm{~km} \mathrm{~s}^{-1} \mathrm{Mpc}^{-1}$ cosmology.

Though there is no obvious trend in either panel of Fig. [1. we apply a formal non-parametric Spearman test for correlations (e.g. Wall \& Jenkins 2003). Given a sample of $N$ data pairs of variables, the two variables are ranked such that $\left(X_{i}, Y_{i}\right)$ are the ranks of the variables for the $i$ th pair $\left(1<X_{i}<N\right.$ and $\left.1<Y_{i}<N\right)$. Then, the Spearman rank correlation coefficient is computed:

$$
r_{s}=1-6 \frac{\sum_{i=1}^{N}\left(X_{i}-Y_{i}\right)^{2}}{N^{3}-N}
$$

with range $0<\left|r_{s}\right|<1$; a high value indicates a significant correlation. The coefficient values we find for the spectral index/luminosity pairs are $r_{s}=0.145$ (BL Lacs) and $r_{s}=-0.109$ (FSRQs) and for the spectral index/redshift pairs are $r_{s}=0.141$ (BL Lacs) and $r_{s}=0.238$ (FSRQs). In all cases, such values of $\left|r_{s}\right|$ or smaller occur by chance more than $10 \%$ of the time.
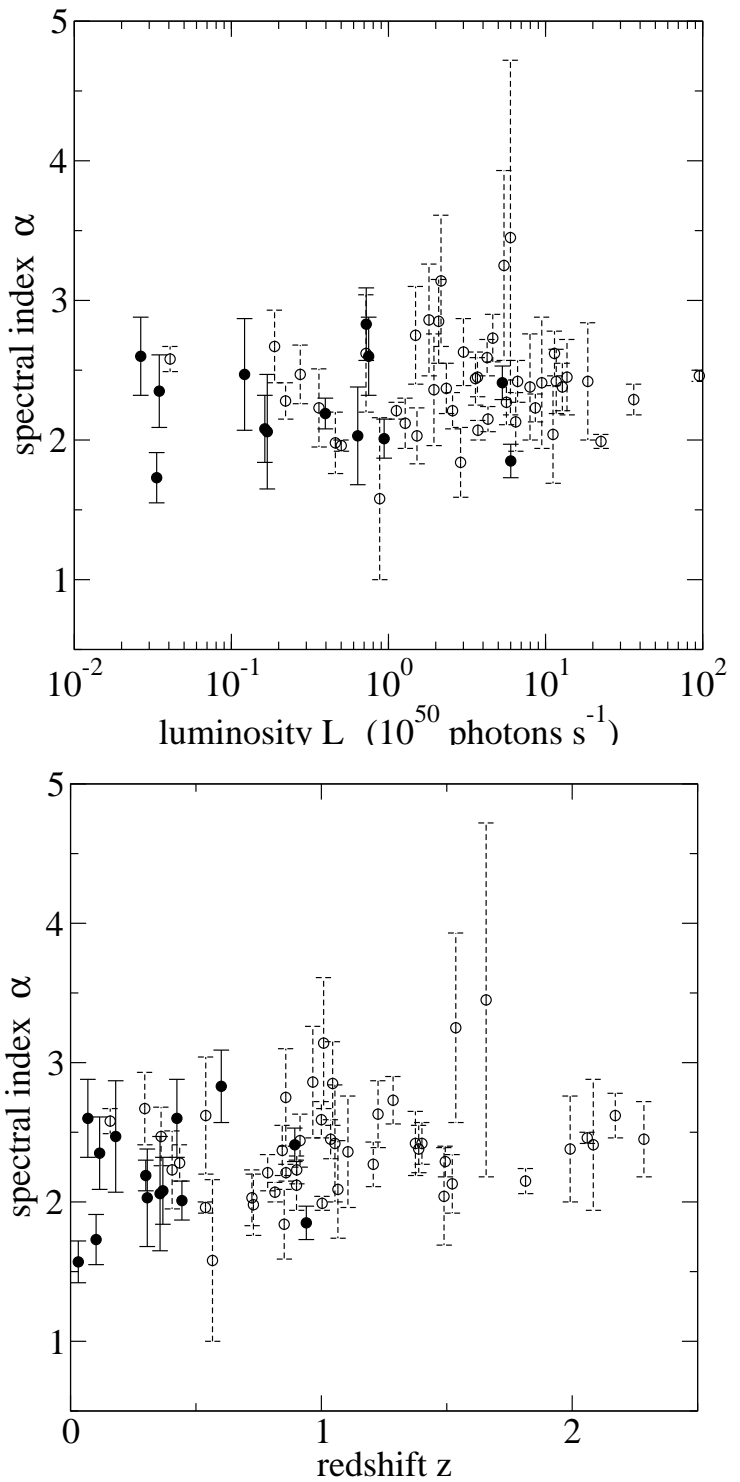

FIG. 1.- Spectral index versus luminosity (upper panel) and versus redshift (lower panel) for BL Lacs (filled circles/solid lines) and FSRQs (open circles/dashed lines).

Therefore, we find no evidence for correlations between spectral index and either redshift or luminosity. Accordingly, we find no evidence for cosmological evolution in the particle acceleration and $\gamma$-ray emisison mechanisms operating in these AGNs, at least in the redshifts under consideration.

\section{THE EFFECT OF MEASUREMENT ERRORS}

We now turn our attention to the reconstruction of the ISID of blazars using measurements of the spectral indices for individual members of the blazar population. We assume that the ISID (probability that a randomly selected blazar has a true spectral index between $\alpha$ and $\alpha+d \alpha)$ can be adequately described by a Gaussian,

$$
p_{\text {intrinsic }}(\alpha) d \alpha=\frac{1}{\sqrt{2 \pi \sigma_{0}}} \exp \left[-\frac{\left(\alpha-\alpha_{0}\right)^{2}}{2 \sigma_{0}^{2}}\right] d \alpha .
$$

The spread of the ISID (which is equal to $\sigma_{0}$, and should not be confused with the "error on the mean," the un- 
certainty in our knowledge of $\alpha_{0}$ ) is not necessarily wellapproximated by the spread of the distribution of measured spectral indices. In sampling a distribution with significant errors in individual measurements, the spread of the resulting measured distribution may be dominated by the errors, especially if they are comparable with the width of the ISID. In this case, if the measured SID is used to calculate the spectral shape of the collective emission of unresolved blazars, sources with spectral indices away from the mean would be overestimated and ultimately lead to an overestimate of the curvature of the collective emission spectrum. It is therefore necessary to determine the degree to which the measured SID is error-dominated before we can decide whether it is representative of the ISID.

In the formalism implemented by SS96a, the measured SID is reconstructed by co-adding all of the probability density functions (assuming Gaussian errors) of individual spectral index measurements of blazars in the second EGRET catalog:

$$
p_{\text {observed }}(\alpha) d \alpha=\frac{1}{N} \sum_{i=1}^{N} \frac{1}{\sigma_{i} \sqrt{2 \pi}} e^{-\frac{\left(\alpha-\alpha_{i}\right)^{2}}{2 \sigma_{i}^{2}}} d \alpha
$$

where $\alpha_{i}$ is the spectral index of blazar $i, \sigma_{i}$ is the error in measurement of $\alpha_{i}$, and $\mathrm{N}$ is the total number of blazars. ${ }^{5}$ Equation (4) is a good representation of the distribution of measured spectral indices (see Fig. 2, SS96a SIDs represented by solid lines) and has the additional advantage that it does not make any a priori assumptions about the shape of the SID, but it could still be contaminated by measurement errors and as such, might not be a fair representation of the ISID. With this concern, we assess the significance of error in the SID, by performing a simple Monte Carlo test.

We consider separately the samples of confident EGRET BL Lacs and FSRQs assuming that the ISID of each subset has the form of Eq. (3). We take the mean of each sample, $\alpha_{0}$, to be the "trimmed mean" (see e.g. Wilcox 1997) of the sample and the variance $\sigma_{0}^{2}$ to be variable. For later comparison, we also calculate, the "trimmed variance," $\sigma_{t}^{2}$, of each population. We calculate $\alpha_{0}$ and $\sigma_{t}^{2}$ by trimming the top and bottom five percent of the combined populations. In this way, we obtain for the BL Lacs: $\alpha_{0}=2.20$ and $\sigma_{t}=0.33$; and for the FSRQs: $\alpha_{0}=2.39$ and $\sigma_{t}=0.22$.

We perform a number of "mock observations" from each ISID equal to the number of corresponding objects EGRET detected, with a randomly chosen 3EG uncertainty for each object, and calculate the trimmed variances of each set. If some assumed ISID spread, sampled with EGRET uncertainties, more frequently results in simulated trimmed variances smaller (larger) than that of the corresponding EGRET dataset, then it is most likely too small (large) compared with the ISID spread occurring in nature. The spread of the ISIDs of BL Lacs and FSRQs occurring in nature should be comparable to the spread for which the simulated datasets most frequently have trimmed variances comparable to those of

5 SS96a also accounted for the different blazar states (flaring/quiescent) by shifting this distribution (toward harder/softer indices), but they considered BL Lacs and FSRQs as a single population.

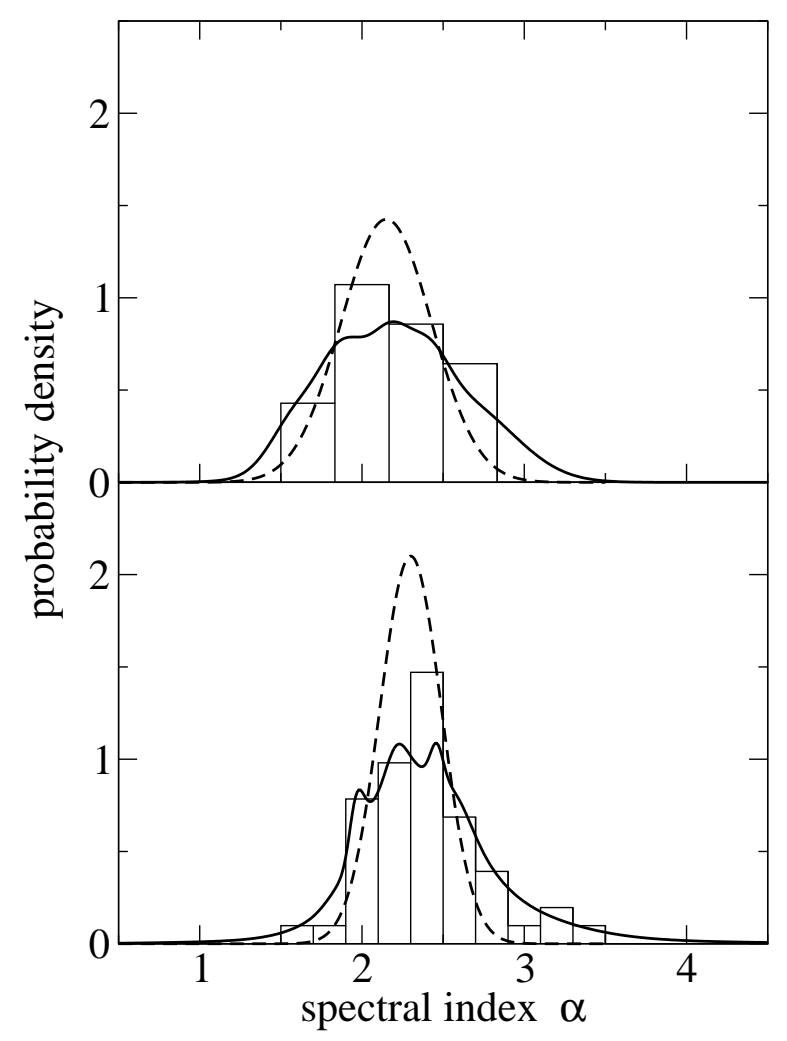

Fig. 2.- Top: Histogram of spectral indices of 3EG BL Lacs with SS SID (solid) overlayed. Bottom: Same for the 3EG FSRQs. The dashed lines represent the maximum-likelihood Gaussians for the same sets (see 4 ).

the EGRET datasets. Figure 3 demonstrates this comparison between the trimmed variances of the EGRET set and the simulated sets. The fraction of simulated sets with trimmed variance greater than that of the corresponding EGRET set is plotted against the spread of the parent ISID. The median ISID spread is equal to 0.27 for the BL Lacs and 0.20 for FSRQs. If we were to fit a Gaussian to the SS96a prescription for the SID, we would obtain a standard deviation of 0.46 in the case of BL Lacs and 0.36 in the case of FSRQs - almost twice the preferred values of the Monte-Carlo analysis. Hence, we conclude that the SS96a prescription does overestimate significantly the spread of the ISID.

\section{THE ISID OF EGRET BLAZARS - A LIKELIHOOD APPROACH}

Having shown that measurement errors can significantly contaminate the determination of the ISID, we now employ a likelihood analysis which allows us to explicitly account for these errors and constrain the parameters of the ISID. Explicitly accounting for measurement errors is necessary as ignoring them may not simply increase the uncertainty in our estimated parameters but lead to incorrect parameter inferences (e.g. Loredo 2004).

Given a set of parameters $x_{i}$, which define a statistical distribution, and a dataset $y_{j}$, the probability of $x_{i}$ having certain values given the data is proportional to the probability of measuring $y_{j}$ given those values of $x_{i}$ (the likelihood) times the probability that $x_{i}$ has those partic- 


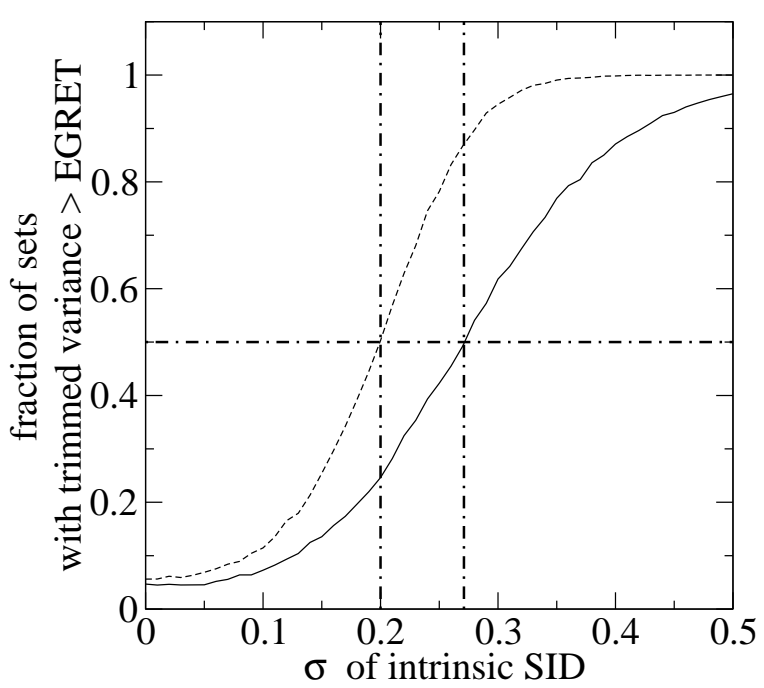

FIG. 3.- Fraction of sets for which the trimmed variance of the set with errors is greater than that of the EGRET set without errors for sets with the same number of objects as confident BL Lacs (solid line) or FSRQs (dotted line). The dot-dashed lines indicate the median "true spread" of the simulated samples, which is equal to 0.27 for BL Lacs and 0.2 for FSRQs.

ular values before measurements are taken (the prior):

$$
P\left(x_{i} \mid y_{j}\right) \propto P\left(x_{i}\right) \times \mathcal{L}\left(y_{j} \mid x_{i}\right) .
$$

If the prior is flat (constant for all values of $x_{i}$ ), maximizing the likelihood allows us to determine the most probable values for the parameters $x_{i}$ (see, e.g., Lee 1989).

In our analysis, we assume that both the ISID and the errors are Gaussian. The parameters we wish to calculate are the mean spectral index, $\alpha_{0}$, and spread, $\sigma_{0}$. Without error, the likelihood of measuring a spectral index $\alpha$ given a Gaussian ISID with a mean of $\alpha_{0}$ and a spread of $\sigma_{0}$ would be $l=\exp \left[-\left(\alpha-\alpha_{0}\right)^{2} / 2 \sigma_{0}^{2}\right] / \sqrt{2 \pi} \sigma_{0}$. To include measurement error, we need to distinguish between the true spectral index of an object, $\alpha$ (which is unknown and is therefore a nuisance parameter over which we need to marginalize), and its measured spectral index, $\alpha_{j}$. Then, the likelihood for a single spectral index measurement becomes

$l_{j}=\int_{-\infty}^{\infty} d \alpha \frac{\exp \left[-\left(\alpha-\alpha_{j}\right)^{2} /\left(2 \sigma_{j}^{2}\right)\right]}{\sqrt{2 \pi} \sigma_{j}} \frac{\exp \left[-\left(\alpha-\alpha_{0}\right)^{2} /\left(2 \sigma_{0}^{2}\right)\right]}{\sqrt{2 \pi} \sigma_{0}}$

where the subscript denotes blazar $j$. If we have $N$ such independent spectral index measurements, then the overall likelihood becomes $\mathcal{L}=\prod_{j=1}^{N} l_{j}$ or, omitting constant normalization factors,

$$
\mathcal{L}=\left(\prod_{j=1}^{N} \frac{1}{\sqrt{\sigma_{0}^{2}+\sigma_{j}^{2}}}\right) \exp \left[-\frac{1}{2} \sum_{j=1}^{N} \frac{\left(\alpha_{j}-\alpha_{0}\right)^{2}}{\sigma_{0}^{2}+\sigma_{j}^{2}}\right] .
$$

The maximum-likelihood mean and spread of the ISID can be found by maximizing the likelihood; hence, by simultaneously solving the equations (for derivation, see Appendix)

$$
\alpha_{0}=\left(\sum_{j=1}^{N} \frac{\alpha_{j}}{\sigma_{0}^{2}+\sigma_{j}^{2}}\right)\left(\sum_{j=1}^{N} \frac{1}{\sigma_{0}^{2}+\sigma_{j}^{2}}\right)^{-1},
$$

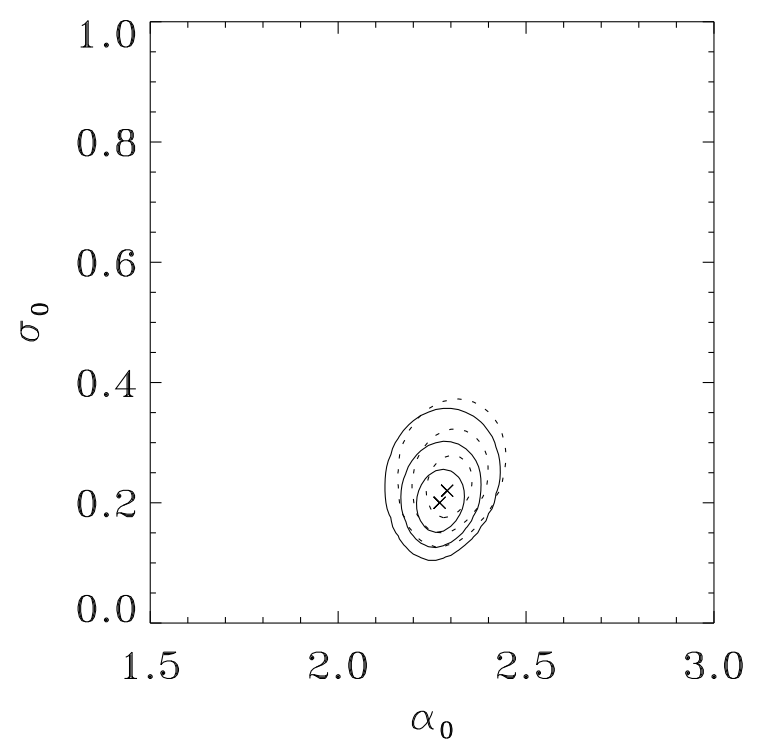

FIG. 4. - Likelihood function contours for the Mattox 2001 (solid lines) and the 3EG (dashed lines) confident blazar samples. The $1 \sigma, 2 \sigma$, and $3 \sigma$ contours we plot throughout this paper represent equal-likelihood contours which include $68 \%, 95.5 \%$ and $99.7 \%$ of the total volume under the likelihood surface. The $\mathrm{x}$ and $\mathrm{y}$ axes represent the mean $\left(\alpha_{0}\right)$ and spread $\left(\sigma_{0}\right)$ of the ISID respectively. The maximum-likelihood parameters of each ISID are denoted by $\times$.

and

$$
\sum_{j=1}^{N} \frac{1}{\sigma_{0}^{2}+\sigma_{j}^{2}}=\sum_{j=1}^{N} \frac{\left(\alpha_{j}-\alpha_{0}\right)^{2}}{\left(\sigma_{0}^{2}+\sigma_{j}^{2}\right)^{2}} .
$$

In Fig. 4, we plot likelihood contours for the confident blazar set of Mattox 2001 (solid lines) and of the 3EG (dashed lines). The maximum-likelihood parameters for the ISID in the case of the Mattox set are $\alpha_{0}=2.27$ and $\sigma_{0}=0.20$, and those for the 3EG set are $\alpha_{0}=2.29$ and $\sigma_{0}=0.22$. As we can see in Fig. 4, the likelihood functions of the two sets are consistent with each other, which shows that our analysis is not sensitive to the inclusion or exclusion of a few members.

\section{BL LACS VS FSRQS: DO THEIR ISIDS DIFFER?}

Gamma-ray-loud BL Lacs and FSRQs have different properties in the $\mathrm{GeV}$ energy range (e.g. different variability properties, Vercellone et al 2004; different mean spectral properties, Mukherjee et al 1997, Pohl et al 1997; different redshift distributions and possibly different luminosity functions, Mücke \& Pohl 2000, Dermer 2007). It is therefore reasonable to test whether the maximumlikelihood ISID for the BL Lacs and FSRQs differ at a statistically significant level.

We apply the analysis of $\$ 4$ to the sets of BL Lacs and FSRQs of the 3EG catalog. Fig. 5 shows the likelihood function contours for these populations. The ISID parameters are found to be $\alpha_{0}=2.15, \sigma_{0}=0.28$ for $\mathrm{BL}$ Lacs and $\alpha_{0}=2.3, \sigma_{0}=0.19$ for FSRQs. The likelihood functions of the two populations do indicate a marginal, $1 \sigma$ separation. This possible spectral differentiation between the two populations, if true, will be confidently confirmed by GLAST data (see 87). The maximumlikelihood $\alpha_{0}$ 's are close to the trimmed means of each 


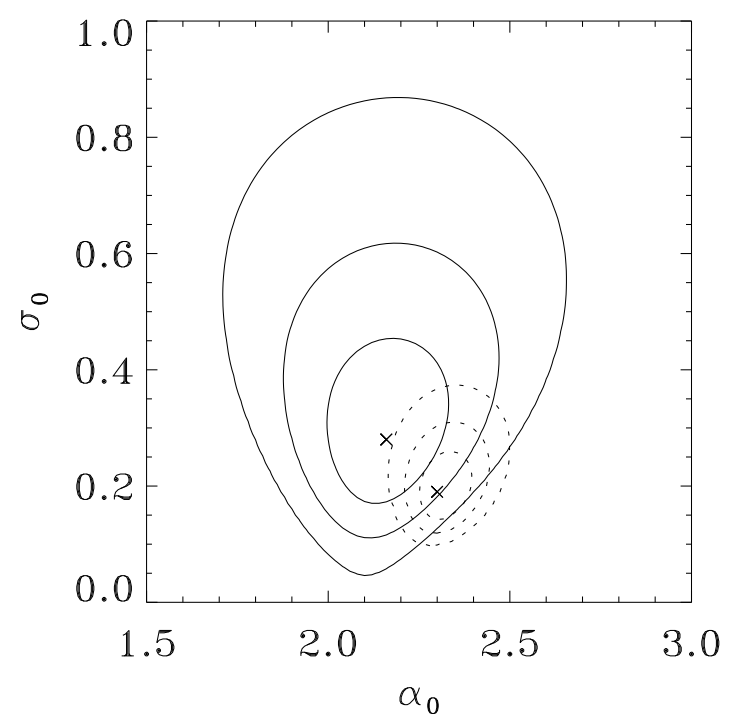

FIG. 5.- Likelihood function contours (as in Fig. 4) for 3EG BL Lacs (solid lines) and FSRQs (dotted lines).

dataset, while the maximum-likelihood $\sigma_{0}$ 's are almost identical to the median "true spread" for each set, as determined by the Monte Carlo test outlined in $\$ 3$ indicating the consistency in the results of the two analyses. Our maximum-likelihood Gaussians for BL Lacs and FSRQs are overplotted on Fig. 2 with the dashed lines.

\section{BLAZAR VARIABILITY AND THE MEASURED SPECTRAL INDEX}

Though a blazar may spend most of its time in a quiescent state, it can undergo periods of flaring during which its flux is significantly enhanced, up to an order of magnitude (e.g. McLaughlin et al. 1996; Mukherjee et al. 1997; Nolan et al. 2003; Vercellone et al. 2004). This increase in flux may introduce a detection selection effect for fainter blazars favoring the flaring state. On the other hand, blazars are more likely to be pointed at during quiescence since they spend more time in the quiescent state. The spectral index of a blazar is determined using the integrated EGRET maps which can include photons from both states, thus complicating the determination of the ISID: it is unclear whether the spectral index distribution of blazars determined from EGRET data is representative of blazars in their flaring or quiescent state. This distinction is important because it has been suggested that the blazar spectrum might change during the flaring state. If such a trend is indeed present and most photons involved in EGRET blazar detections come from blazars in the flaring state, then the derivation of the SID will be biased towards spectral indices representative of flaring blazars (as was assumed in SS96a).

Determining whether a blazar is in a flaring or quiescent state during a certain viewing period is made complicated by low photon statistics. During the long exposure necessary to detect a blazar, the source could be in both states, and the duty cycle is largely unknown (Vercellone et al. 2004). Furthermore, the source identification and flux estimation for different viewing periods and for the cumulative map (from which the spectral index is derived) is done by separate statistical processing of each map, and as a result, the photon counts from the individual viewing periods don't add up to those of the cumulative map. With these uncertainties in mind, we approach the problem using a simple recipe.

In a manner similar to that of Vercellone et al. (2004), we classify each individual viewing period of a confident AGN (excluding periods for which only flux upper limits were quoted) as a "mostly" flaring period if the flux of the blazar in the particular viewing period, $F_{\mathrm{VP}}$, is $>\mathcal{F} \times F_{\mathrm{P} 1234}$ (where $\mathcal{F}>1$ is some constant enhancement factor) and if the error on the measurement of $F_{\mathrm{VP}}$ is less than the difference between the $F_{\mathrm{VP}}$ and $F_{\mathrm{P} 1234}$; otherwise, we classify it as a "mostly" quiescent period. As in Vercellone et al. (2004), and in Jorstad et al. (2001), we consider the conventional case ${ }^{6}$ of $\mathcal{F}=1.5$. We also consider the case of $\mathcal{F}=3.75$, chosen as the enhancement factor for which $\sim 75 \%$ of the objects have a flaring fraction below 0.2 .

Once we have classified all of the viewing periods during which a blazar was detected, we add all of the photon counts from the flaring periods to get the number of flaring photons, $N_{f}$, and likewise for the quiescent periods to get the number of quiescent photons, $N_{q}$. We then calculate the "flaring photon fraction," $f=N_{f} /\left(N_{f}+N_{q}\right)$, for every confident blazar.

Figure 6 shows the fraction of blazars with a flaring fraction greater than $f$ as a function of $f$, for both enhancement factors. As expected, the flaring photon fraction depends on our definition of a "flaring state:" the fraction of objects with flaring photon fraction $>f$, is systematically lower for all values of $f$ for the higher value of $\mathcal{F}$. However, even for $\mathcal{F}=1.5$, the median flaring photon fraction is not very high (equal to 0.5 for BL Lacs, and 0.6 for FSRQs, while only $20 \%$ of BL Lacs and $40 \%$ of FSRQs have a flaring photon fraction higher than 0.7 , at which point photons predominantly come from a flaring state). Therefore, the photons that are used to derive individual blazar spectra represent a balanced mix of photons originating in flaring and quiescent states, if not photons that come primarily from the quiescent state (with the quantitative details of this statement depending on the value of the enhancement factor). This analysis suggests that even if spectral indices of blazars do harden considerably during the flaring states for most objects, there is no indication that the photon budget is overly biased towards one variability state. Hence, if a SID is determined from EGRET data, there is no evidence that it would be predominantly representative of the flaring-state or quiescent-state SID.

Qualitatively, this result also has implications for the blazar duty cycle. If the time spent flaring is comparable to the time spent in quiescence, we would expect the number of flaring photons to be significantly greater than the number of quiescent photons, yielding a flaring fraction close to one. This however is not the case even

\footnotetext{
${ }^{6}$ Note that Vercellone et al. (2004) and Jorstad et al. (2001) compared the individual viewing periods with the inverse-uncertaintyweighted mean of fluxes and upper limits of the individual viewing periods. However, this weighted flux is more biased towards higher fluxes than the true long-term average flux of the blazar, as a blazar is preferentially detected in a viewing period when it is flaring (Vercellone et al. 2004). Thus, we systematically identify more "flaring" periods than Vercellone et al. (2004) and Jorstad et al. (2001).
} 


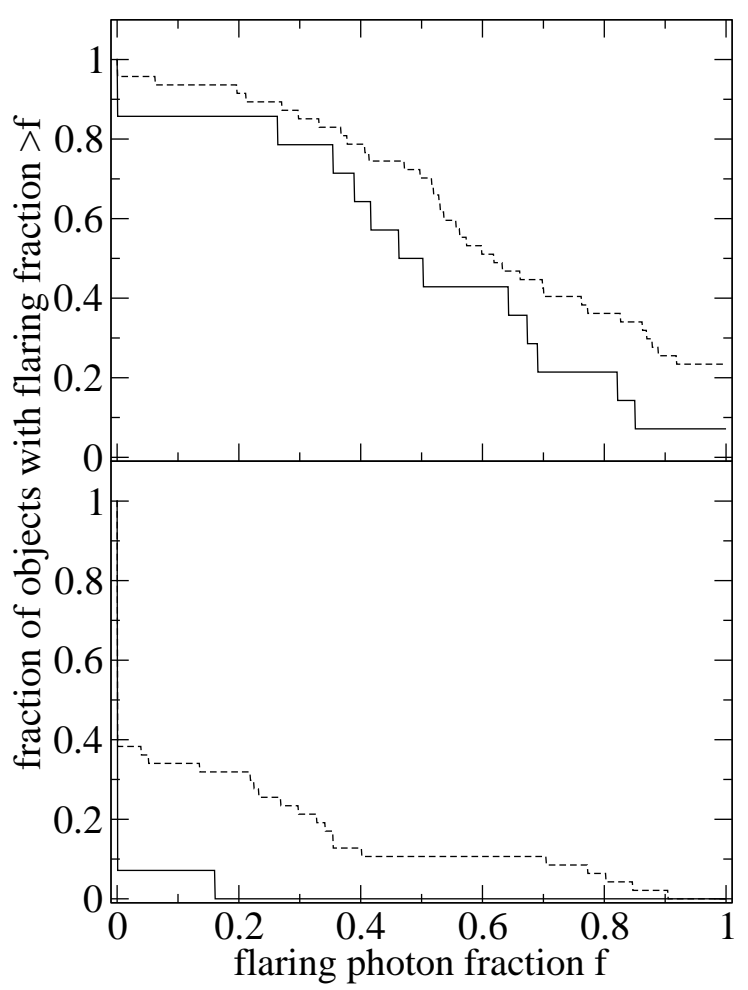

FIG. 6.- Fraction of objects with fraction of flaring photons greater than $f$ as a function of $f$ for $\mathcal{F}=1.5$ (upper panel) and $\mathcal{F}=3.75$ (lower panel) for BL Lacs (solid lines) and FSRQs (dotted lines).

for a lower value of $\mathcal{F}$. Therefore, these blazars must have been viewed more often in their quiescent state.

\subsection{Is there statistical evidence for spectral index hardening during flaring?}

As a first indication for spectral index shift with flaring, we can test for a spectral index/flaring photon fraction correlation. No obvious trend exists but, as in $\$ 2$, we also perform a non-parametric Spearman rank coefficient test, treating the BL Lacs and FSRQs separately, and for $\mathcal{F}=1.5$ and $\mathcal{F}=3.75$.

With the exception of BL Lacs in the $\mathcal{F}=3.75$ case, the data are consistent with uncorrelated variables at the $20 \%$ level. In the case of $\mathcal{F}=3.75$, all BL Lacs but one have $f=0$, which gives a false positive result in the correlation test. However, when we treat the BL Lacs and the FSRQs as a single population, the results are again consistent with uncorrelated variables. A recent systematic reanalysis of EGRET data for all blazars bright enough for time-resolved spectroscopy by Nandikotkur et al. (2007) has also yielded similar results despite using a radically different approach, as no systematic trend of the spectral index with changing flux was found.

A second method to look for a systematic spectral index shift with changing flux is to perform the maximumlikelihood analysis of $\$ 4$ separately for the populations of "mostly flaring" and "mostly quiescent" blazars. The set of "mostly flaring" blazars are the 22 EGRET blazars that have a flaring photon fraction $f>0.7$, for $\mathcal{F}=1.5$. The set of "mostly quiescent" blazars are the 10 EGRET blazars that have flaring photon fractions $f<0.3$. Likelihood function contours for the ISID parameters of these
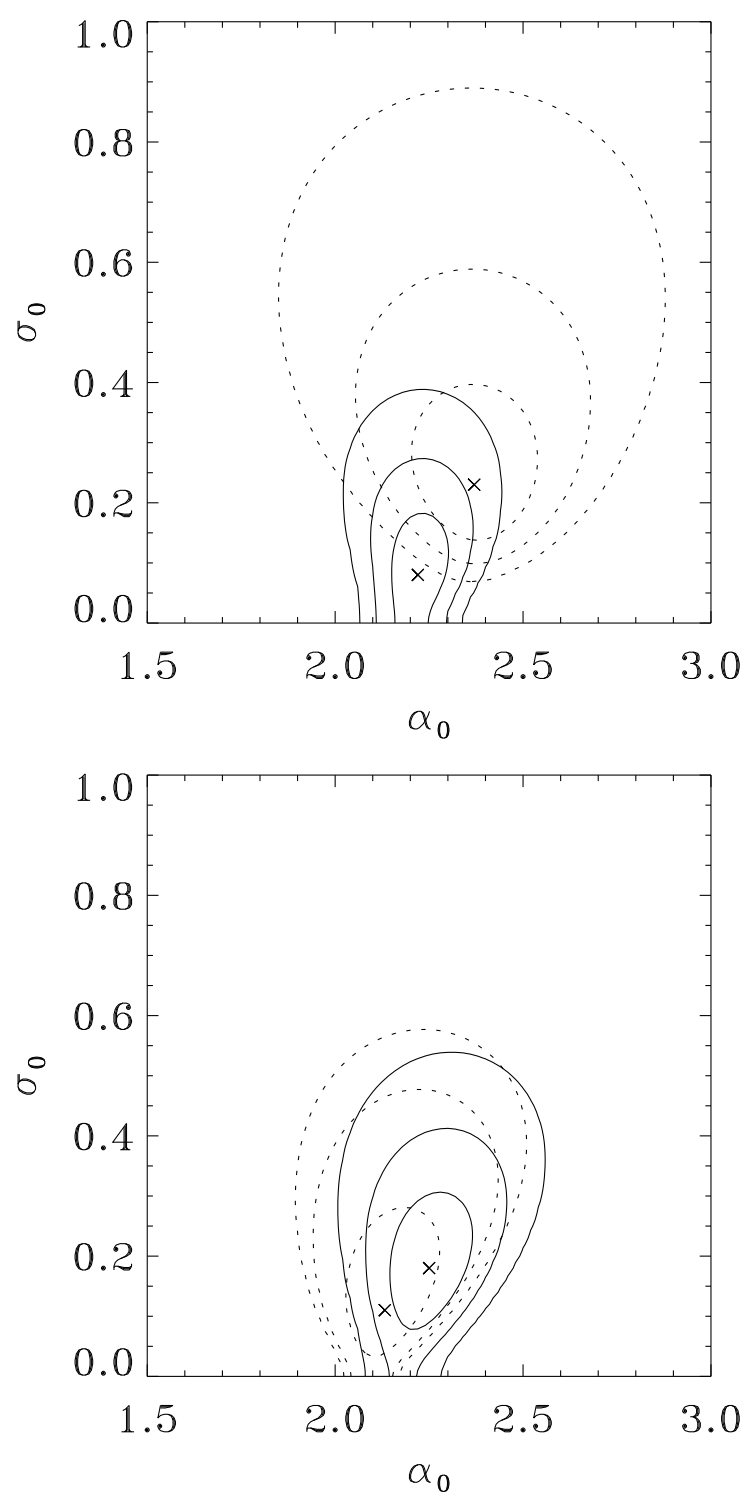

FIG. 7.- Likelihood function contours (as in Fig. 4) for mostly flaring (solid lines) and mostly quiescent (dashed lines) blazars. Upper panel: 3EG blazars; lower panel: simulated EGRET dataset.

two sets are shown in the upper panel of Fig. 7. The ISIDs of the two sets are fully consistent with each other, with maximum-likelihood parameters for the "mostly flaring" blazars $\alpha_{0}=2.25$ and $\sigma_{0}=0.18$, and for the "mostly quiescent" blazars $\alpha_{0}=2.13$ and $\sigma_{0}=0.11$. If anything, the maximum-likelihood ISID of mostly flaring objects peaks at slightly softer indices than that of the mostly quiescent sources (a trend that could be theoretically accomodated if, for example, softer EC emission overtakes an otherwise harder, SSC dominated, spectrum during flaring; see e.g. Böttcher 1999).

In order to determine whether the EGRET data would be sufficient to reveal spectral index hardening with flaring if such a trend does exist, we perform the following test. We assume that blazar spectral indices do indeed harden with flaring, and that their ISIDs differ as suggested by SS96a: they are identical Gaussians with 
$\sigma_{0}=0.20$ and means that are determined by shifting the mean of the combined population, $\alpha_{0}=2.27$ (mean and spread obtained from the maximum-likelihood analysis of the Mattox et al. 2001 set), by -0.05 for the flaring blazars and by +0.2 for quiescent blazars. We then sample this dataset with EGRET uncertainties (as in 93 ) and create a mock EGRET dataset. We apply the same likelihood analysis to these simulated datasets. Likelihood function contours are plotted in the lower panel of Fig. 7. Although the maximum-likelihood analysis correctly identifies the flaring population ISID as being harder than the quiescent population ISID, the separation of the maxima of the likelihood functions has a marginal $1 \sigma$ significance. Additionally, in the EGRET dataset the measurements of the spectral index do not come from pure "flaring" or "quiescent" states, and even the definition of a "flaring" or "quiescent" state is dependent on the selected value of the enhancement factor $\mathcal{F}$. Thus, we conclude that although EGRET data do not seem to indicate a hardening of the spectral index with flaring, they are not sufficient to exclude such a possibility either. However, as we will see in the next section, GLAST data will settle the issue.

\section{PROSPECTS FOR GLAST}

With the launch of GLAST later this year, the number statistics of resolved blazars are expected to improve dramatically. Depending on models used to determine blazar numbers at lower fluxes, GLAST will detect between 1,000 - 10,000 blazars with flux $>2 \times$ $10^{-9}$ photons s ${ }^{-1}$. However, most of these blazars will have fluxes close to the lower end of GLAST sensitivity with individual photon statistics and spectral index uncertainties comparable to those of the EGRET dataset. Thus, the extent to which the GLAST dataset will improve our understanding of the issues discussed in this paper is not immediately obvious.

To assess this question, we construct simulated datasets of spectral indices and spectral index uncertainties for GLAST-detectable blazars. Based on these datasets, we predict by how much GLAST observations will improve the determination of statistical properties of the spectral indices of gamma-ray-loud blazars. For our study, we use the Dermer (2007) gamma-ray luminosity functions for BL Lacs and FSRQs. These luminosity functions are more conservative than those of, e.g., Narumoto \& Totani (2006), Chiang \& Mukherjee (2001) and SS96a as they predict a smaller number of detectable blazars; additionally, they treat BL Lacs and FSRQs separately, enabling us to distinguish between the two populations in our analysis. If GLAST detects more blazars than predicted in Dermer (2007) and assumed here, the statistics will only improve, strengthening the significance of our results.

In assigning measurement uncertainties to the spectral index of each blazar in our simulated datasets, we make use of the strong anticorrelation of spectral index uncertainties in the EGRET dataset with the total number of detected P1234 photons. A single power-law can be fitted to the $3 \mathrm{EG}$ data,

$$
\sigma_{\alpha}=7.0 \times N_{\text {photon }}^{-0.7}
$$

We expect that, despite any differences between the Large Area Telescope (LAT) and EGRET instruments,

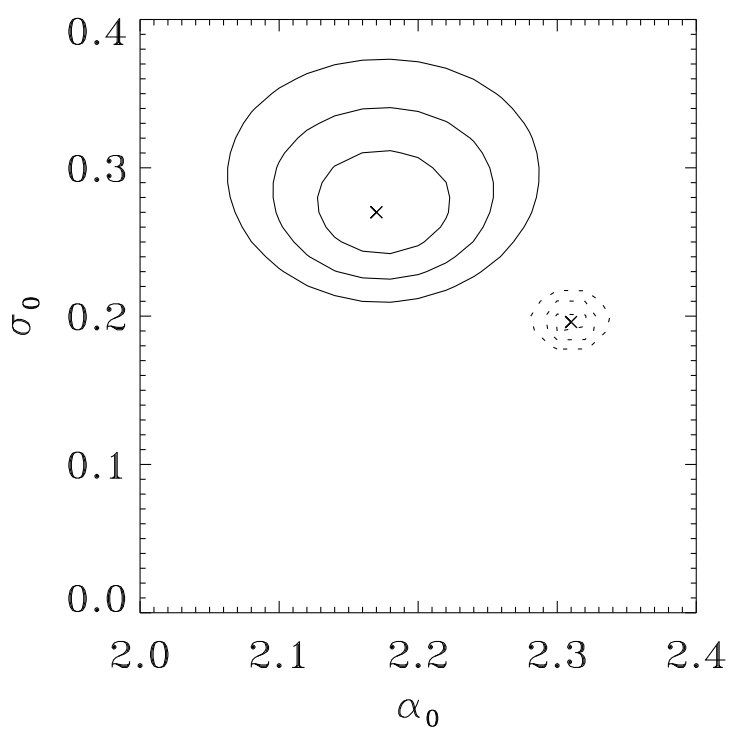

FIG. 8.- Likelihood function contours (as in Fig. 4) for simulated GLAST datasets of BL Lacs (solid lines) and FSRQs (dashed lines)

this empirical relation can also give a rough approximation of the spectral index uncertainty for blazars detected by GLAST.

\subsection{Spectral separation between BL Lacs and FSRQs}

Using simulated GLAST datasets, we examine the possibility that, if BL Lacs and FSRQs are indeed spectrally distinct populations, GLAST observations will be sufficient to separate them with a significance greater than $3 \sigma$. We assume that the ISIDs for BL Lacs and FSRQs are the maximum-likelihood Gaussians determined from EGRET data $\left(\alpha_{0}=2.15\right.$ and $\sigma_{0}=0.28$ for BL Lacs and $\alpha_{0}=2.3$ and $\sigma_{0}=0.19$ for FSRQs). We generate the simulated BL Lac and FSRQ datasets by appropriately sampling these ISIDs with GLAST uncertainties. The number of objects in each set and their fluxes are derived from Fig. 2 of Dermer (2007). From the flux of each object, we derive an approximate number of detectable photons, $N_{\text {photon, } i}=F_{i} \Delta t A$, where $F_{i}$ is the flux of the particular blazar, $\Delta t$ is the estimated exposure time (assuming that any given objects will be in the GLAST field of view for $\sim 20 \%$ of a 2 year campaign), and $A$ is the effective collecting area of the detector $\left(8,000 \mathrm{~cm}^{2}\right.$ for GLAST). The spectral index uncertainty for each object is then derived from Eq. 10.

Performing the analysis outlined in 4 on these datasets, we get the likelihood function contours shown in Fig. 8. The maximum-likelihood parameters of the ISIDs are $\alpha_{0}=2.17$ and $\sigma_{0}=0.27$ for the BL Lacs, and $\alpha_{0}=2.31$ and $\sigma_{0}=0.2$ for the FSRQs, excellent approximations of the assumed underlying ISID parameters. The separation between the two populations is clearly established with significance greater than $3 \sigma$.

\subsection{Spectral separation between flaring and quiescent blazars}

Finally, we investigate the possibility that GLAST observations will be sufficient to determine whether flaring and quiescent blazars are indeed spectrally distinct. We 
construct simulated GLAST datasets to test two scenarios: that the ISIDs of flaring and quiescent blazars have the same shape, but flaring blazars are shifted towards harder indices by 0.25 (as in 96.1 ); and that flaring and quiescent blazars have ISIDs with parameters equal to the maximum-likelihood parameters yielded by the analysis of the true EGRET dataset (note that in this case the flaring blazars are somewhat softer than quiescent blazars).

As there are many more blazars in this analysis, we can account for the disparities between the flaring fractions for bright and faint blazars by calculating (from EGRET data) the fractions of bright and faint objects that are mostly flaring $(f>0.7)$ or mostly quiescent $(f<0.3)$; thereby, more carefully determining the numbers of mostly flaring and mostly quiescent blazars. We assign true spectral indices as in 6.1 and spectral index uncertainties based on the flux as in $\$ 7.1$, treating BL Lacs and FSRQs as a single population.

We then apply the same likelihood analysis to these simulated datasets. Their likelihood function contours are plotted in Fig. 9, Although the expected partial contamination of any single state with both flaring and quiescent photons may weaken this result, likelihood functions for the flaring and the quiescent blazars are well separated with a significance greater than $3 \sigma$ in both scenarios, strongly suggesting that the GLAST data will reveal any systematic change of spectral index with flaring, if present.

\section{DISCUSSION AND SUMMARY}

In this work, we have extensively studied possible trends in the spectral behavior of EGRET blazars, and have attempted to reconstruct the blazar ISID from EGRET observations, explicitly accounting for the large measurement errors arising from low-photon statistics. Additionally, we have identified still open questions on which progress is expected to be made once GLAST observations become available, and we have estimated the extent of the expected improvement. Our conclusions can be summarized in the following statements:

(a) We have investigated the possibility that source evolution could interfere with the application of our ISIDs to unresolved blazars. We have found no evidence for correlations between spectral index and redshift or between spectral index and luminosity in EGRET data. Therefore, we have concluded that there are no indications for spectral index evolution in EGRET data.

(b) We have used a Monte Carlo test to study whether measurement errors in individual spectral indices severely contaminate the observed SID. We found that measurement errors can indeed have a profound effect and need to be accounted for properly. This will also be the case for GLAST data, as most of the newly detected blazars will suffer from similar low-photon statistics as with the EGRET blazars. We have performed a likelihood analysis which explicitly accounts for significant and unequal errors of measurement, and we found a most likely Gaussian ISID for the population of EGRET blazars with a mean of 2.27 and a spread of 0.20 , significantly narrower than the observed SID.

(c) We have derived separate ISIDs for BL Lacs and FSRQs finding that FSRQs are well represented by a Gaussian with mean of 2.3 and spread of 0.19 , while the
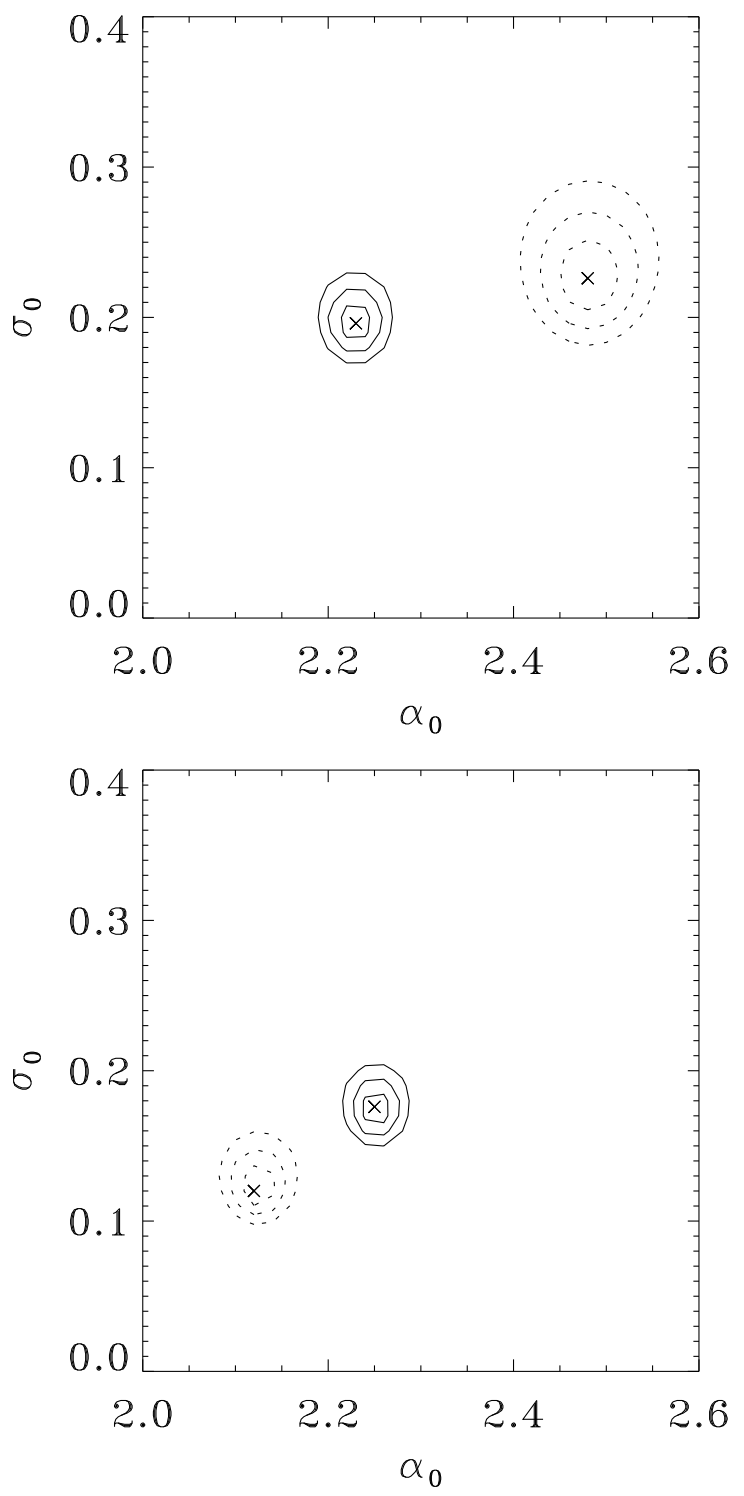

FIG. 9.- Likelihood function contours as in Fig. 7 for simulated GLAST data. Upper panel: likelihood contours assuming flaring blazars are harder; lower panel: likelihood contours assuming flaring and quiescent blazars ISID with parameters determined in the analysis of the EGRET simulated variability dataset.

BL Lacs are better represented by a Gaussian with mean of 2.15 and spread of 0.28. Based on EGRET data, the significance of the spectral separation of the two populations is marginal. We determined whether each spectral index of the EGRET confident blazars was more representative of the flaring or quiescent state by calculating the flaring photon fraction for each object. We performed statistical tests for spectral index hardening and found no evidence for this effect. We calculated separate maximum-likelihood ISIDs for mostly flaring and mostly quiescent objects finding no evidence for a systematic separation of the two populations. However, we also showed that such a separation can neither be excluded based on EGRET data. This result is consistent with the recent findings of Nandikotkur et al. (2007) who studied spectral index shifting during flaring on an objectto-object basis and also found no systematic trend. Ul- 
timately, the question of hardening will be addressed by GLAST through more confident measurements of spectral indices and an increased ability for time-resolved spectroscopy (Dermer \& Dingus 2004) allowing confident measurements of spectral indices for separate variability states of blazars.

(d) We have made explicit estimates about the improvement of our statistical analysis once GLAST data become available finding that, with the detection of many more blazars, GLAST will be able to separate with significance $>3 \sigma$ the ISIDs of BL Lacs and FSRQs, if these populations are indeed spectrally distinct. Similarly, we have examined two different scenarios for a possible systematic spectral shift during flaring finding that in both cases, GLAST would be able to distinguish between the two ISIDs at $>3 \sigma$.

The maximum-likelihood analysis presented in this work is therefore a useful tool in maximizing the information that can be obtained from the high-uncertainty measurements of spectral indices in the GeV band, where photon statistics for most objects are very low. Addition- ally, it can provide estimates for both the mean and the spread of the underlying, intrinsic distribution(s) of spectral indices of $\gamma$-ray emitters. Both of these aspects of the ISID(s) are not only important tests for $\gamma$-ray emission models for blazars, but also essential inputs in determining the spectral shape of the diffuse emission from unresolved blazars (Pavlidou \& Venters 2007).

We gratefully acknowledge enlightening discussions with Markus Böttcher, Chuck Dermer, Brian Fields, Demos Kazanas, Zhaoming Ma, Reshmi Mukherjee, Angela Olinto, Martin Pohl, Jennifer Siegal-Gaskins, and insightful comments by Kostas Tassis that improved this paper. T.M.V. would also like to thank the members of the Laboratoire d'AstroParticule et Cosmologie of the Université de Paris 7 for their hospitality and camaraderie during the final stages of this work. This work was supported by the Kavli Institute for Cosmological Physics through the grant NSF PHY-0114422. T.M.V. was supported by an NSF Graduate Research Fellowship.

\section{REFERENCES}

Blandford, R. D., \& Konigl, A. 1979, ApJ, 232, 34

Brecher, K., \& Burbidge, G. R. 1972, ApJ, 174, 253

Boettcher, M. 1999, ApJ, 515, L21

Boettcher, M. 2006, ArXiv Astrophysics e-prints, arXiv:astro-ph/0608713 Petrosian, V. 1995, ApJ, 452, 156

Chiang, J., \& Mukherjee, R. 1998, ApJ, 496, 752

Dar, A., \& De Rújula, A. 2001, MNRAS, 323, 391

Dermer, C. D., \& Dingus, B. L. 2004, New Astronomy Review, 48, 537

Dermer, C. D. 2007, ApJ in print (arXiv:astro-ph/0605402)

Giommi, P., Colafrancesco, S., Cavazzuti, E., Perri, M., \& Pittori, C. 2006 , A\&A, 445,843

Hartman, R. C., et al. 1999, ApJS, 123, 79

Jorstad, S. G., Marscher, A. P., Mattox, J. R., Aller, M. F., Aller, H. D., Wehrle, A. E., \& Bloom, S. D. 2001, ApJ, 556, 738

Kazanas, D., \& Perlman, E. 1997, ApJ, 476, 7

Keshet, U., Waxman, E., \& Loeb, A. 2004, JCAP, 4, 6

Kneiske, T. M., Mannheim, K. 2005, Proceedings of the 29th International Cosmic Ray Conference, 4, 1

Lee, P. 1989, Bayesian statistics: an introduction (New York: Oxford University Press)

Loredo, T. J. 2004, American Institute of Physics Conference Series, 735, 195

McLaughlin, M. A., Mattox, J. R., Cordes, J. M., \& Thompson, D. J. 1996, ApJ, 473, 763

Mücke, A., Pohl, M., Kanbach, G., Reich, P., Reich, W., \& Schlickeiser, R. 1996, IAU Symp. 175: Extragalactic Radio Sources, 175, 285

Mücke, A., \& Pohl, M. 2000, MNRAS, 312, 177

Mukherjee, R., et al. 1996, ApJ, 470, 831

Mukherjee, R., et al. 1997, ApJ, 490, 116

Mukherjee, R., \& Chiang, J. 1999, Astroparticle Physics, 11, 213
Nandikotkur, G., Jahoda, K. M., Hartman, R. C., Mukherjee, R., Sreekumar, P., Böttcher, M., Sambruna, M. \& Swank, J. H. 2007, ApJ, 657, 706

Narumoto, T., \& Totani, T. 2006, ApJ, 643, 81

Nolan, P. L., Tompkins, W. F., Grenier, I. A., \& Michelson, P. F. 2003, ApJ, 597, 615

Padovani, P., Ghisellini, G., Fabian, A. C., \& Celotti, A. 1993, MNRAS, 260, L21

Pavlidou, V. \& Venters, T. M. 2007, in preparation

Pavlidou, V., \& Fields, B. D. 2002, ApJ, 575, L5

Pohl, M., Hartman, R. C., Jones, B. B., \& Sreekumar, P. 1997, A\&A, 326, 51

Salamon, M. H., \& Stecker, F. W. 1994, ApJ, 430, L21

Salamon, M. H., \& Stecker, F. W. 1998, ApJ, 493, 547

Stecker, F. W., Salamon, M. H., \& Malkan, M. A. 1993, ApJ, 410, L71

Stecker, F. W., \& Salamon, M. H. 1996a, ApJ, 464, 600

Stecker, F. W., \& Salamon, M. H. 1996b, Physical Review Letters, 76,3878

Stecker, F. W. \& Salamon, M. H. 1999, Ap\&SS, 261, 327, arXiv:astro-ph/9909157

Strong, A. W., Moskalenko, I. V., \& Reimer, O. 2004, ApJ, 613, 956

Urry, C. M., \& Padovani, P. 1995, PASP, 107, 803

Vercellone, S., Soldi, S., Chen, A. W., \& Tavani, M. 2004, MNRAS, 353,890

von Montigny, C., et al. 1995, ApJ, 440, 525

Wall, J. V., \& Jenkins, C. R. 2004, Practical Statistics for Astronomers (New York: Cambridge University Press)

Wilcox, R. R. 1997, Introduction to Robust Estimation and Hypothesis Testing (San Diego, CA: Academic Press)

\section{APPENDIX}

\section{DERIVATION AND MAXIMIZATION OF LIKELIHOOD FUNCTION FOR THE ISID PARAMETERS}

As discussed in 93 , the likelihood of observing $N$ spectral indices $a_{j}(j=1, \ldots, N)$ with individual measurement uncertainties $\sigma_{j}(j=1, . ., N)$ (where the $\sigma_{j}$ are assumed Gaussian) if the ISID is Gaussian with mean $\alpha_{0}$ and variance $\sigma_{0}$ is $\mathcal{L}=\prod_{j=1}^{N} l_{j}$, with

$$
l_{j}=\int_{a=-\infty}^{\infty} \frac{1}{\sigma_{j} \sqrt{2 \pi}} \exp \left[-\frac{\left(\alpha-\alpha_{j}\right)^{2}}{2 \sigma_{j}^{2}}\right] \frac{1}{\sigma_{0} \sqrt{2 \pi}} \exp \left[-\frac{\left(\alpha-\alpha_{0}\right)^{2}}{2 \sigma_{0}^{2}}\right] d \alpha
$$


Defining

$$
A_{j}=\sigma_{0}^{2}+\sigma_{j}^{2}, \quad B_{j}=\frac{\sigma_{0}^{2} \alpha_{j}+\sigma_{j}^{2} \alpha_{0}}{\sigma_{0}^{2}+\sigma_{j}^{2}}, \quad C_{j}=\frac{\sigma_{j}^{2} \sigma_{0}^{2}\left(\alpha_{j}-\alpha_{0}\right)^{2}}{\sigma_{0}^{2}+\sigma_{j}^{2}},
$$

we can rewrite the exponentials in Eq. (A1) by completing the square as

$$
\exp \left[-\frac{\left(\alpha-\alpha_{j}\right)^{2}}{2 \sigma_{j}^{2}}-\frac{\left(\alpha-\alpha_{0}\right)^{2}}{2 \sigma_{0}^{2}}\right]=\exp \left[-\frac{A_{j}\left(\alpha-B_{j}\right)^{2}+C_{j}}{2 \sigma_{0}^{2} \sigma_{j}^{2}}\right] .
$$

Substituting this in Eq. (A1) and performing the integration we obtain

$$
l_{j}=\exp \left[-\frac{C_{j}}{2 \sigma_{0}^{2} \sigma_{j}^{2}}\right] \frac{1}{\sqrt{2 \pi A_{j}}} .
$$

The likelihood function then becomes

$$
\mathcal{L}\left(\alpha_{0}, \sigma_{0}\right)=\prod_{j=1}^{N} l_{j}=\left(\prod_{j=1}^{N} \frac{1}{\sqrt{2 \pi\left(\sigma_{0}^{2}+\sigma_{j}^{2}\right)}}\right) \exp \left[-\frac{1}{2} \sum_{j=1}^{N} \frac{\left(\alpha_{j}-\alpha_{0}\right)^{2}}{\sigma_{0}^{2}+\sigma_{j}^{2}}\right] .
$$

Local maxima of this function will satisfy

$$
\frac{\partial \mathcal{L}}{\partial \alpha_{0}}=0, \quad \frac{\partial \mathcal{L}}{\partial \sigma_{0}}=0
$$

Equation (A5) implies

$$
\frac{\partial \mathcal{L}}{\partial \alpha_{0}}=\left(\prod_{j=1}^{N} \frac{1}{\sqrt{2 \pi\left(\sigma_{0}^{2}+\sigma_{j}^{2}\right)}}\right) \exp \left[-\frac{1}{2} \sum_{j=1}^{N} \frac{\left(\alpha_{j}-\alpha_{0}\right)^{2}}{\sigma_{0}^{2}+\sigma_{j}^{2}}\right] \sum_{j=1}^{N} \frac{\alpha_{j}-\alpha_{0}}{\sigma_{0}^{2}+\sigma_{j}^{2}},
$$

therefore the first of Eqs. (A6) becomes

$$
\alpha_{0}=\left(\sum_{j=1}^{N} \frac{\alpha_{j}}{\sigma_{0}^{2}+\sigma_{j}^{2}}\right)\left(\sum_{j=1}^{N} \frac{1}{\sigma_{0}^{2}+\sigma_{j}^{2}}\right)^{-1} .
$$

Similarly,

$$
\frac{\partial \mathcal{L}}{\partial \sigma_{0}}=\exp \left[-\frac{1}{2} \sum_{j=1}^{N} \frac{\left(\alpha_{j}-\alpha_{0}\right)^{2}}{\sigma_{0}^{2}+\sigma_{j}^{2}}\right]\left(\prod_{j=1}^{N} \sqrt{\frac{1}{2 \pi\left(\sigma_{0}^{2}+\sigma_{j}^{2}\right)}}\right) \sigma_{0}\left[-\sum_{j=1}^{N} \frac{1}{\sigma_{0}^{2}+\sigma_{j}^{2}}+\sum_{j=1}^{N} \frac{\left(\alpha_{j}-\alpha_{0}\right)^{2}}{\left(\sigma_{0}^{2}+\sigma_{j}^{2}\right)^{2}}\right],
$$

where we have used the identity

$$
\frac{\partial}{\partial \sigma_{0}} \prod_{j=1}^{N} \frac{1}{\sqrt{\sigma_{0}^{2}+\sigma_{j}^{2}}}=-\sigma_{0}\left(\prod_{j=1}^{N} \frac{1}{\sqrt{\sigma_{0}^{2}+\sigma_{j}^{2}}}\right)\left(\sum_{j=1}^{N} \frac{1}{\sigma_{0}^{2}+\sigma_{j}^{2}}\right)
$$

which is straight-forward to prove by induction. Therefore, the second of Eqs. (A6) becomes

$$
\sum_{j=1}^{N} \frac{1}{\sigma_{0}^{2}+\sigma_{j}^{2}}=\sum_{j=1}^{N} \frac{\left(\alpha_{j}-\alpha_{0}\right)^{2}}{\left(\sigma_{0}^{2}+\sigma_{j}^{2}\right)^{2}}
$$

